\title{
Strategies for achieving intense single-cycle pulses with in-line post-compression setups
}

\author{
Francisco Silva, ${ }^{1,2}$ Benjamín Alonso, ${ }^{3}$ (i) Warein Holgado, ${ }^{3,5}$ (i) Rosa Romero, ${ }^{1,2}$ Julio San Román, ${ }^{3}$ \\ Enrique Conejero Jarque, ${ }^{3}$ Hans Koop, ${ }^{4}$ Vladimir Pervak, ${ }^{4}$ Helder $_{\text {Crespo, }}{ }^{2,3}$ (1) and Í̃̃Igo J. Sola ${ }^{3, *}$ (1) \\ 'Sphere Ultrafast Photonics, S.A., Parque de Ciência e Tecnologia da Universidade do Porto, R. do Campo Alegre 1021, \\ Edifício FC6, 4169-007 Porto, Portugal \\ ${ }^{2}$ IFIMUP-IN and Departamento de Física e Astronomia, Universidade do Porto, Rua do Campo Alegre 687, 4169-007 Porto, Portugal \\ ${ }^{3}$ Grupo de Aplicaciones del Láser y Fotónica (ALF), Departamento de Física Aplicada, University of Salamanca, \\ Pl. de la Merced s/n, E-37008 Salamanca, Spain \\ ${ }^{4}$ UltraFast Innovations GmbH, Am Coulombwall 1, 85748 Garching, Germany \\ ${ }^{5}$ Currently at Centro de Láseres Pulsados, C/Adaja, 8, 37185 Villamayor de la Armunia, Spain \\ *Corresponding author: ijsola@usal.es
}

Received 20 November 2017; revised 13 December 2017; accepted 14 December 2017; posted 14 December 2017 (Doc. ID 313694 ); published 15 January 2018

Intense few- and single-cycle pulses are powerful tools in different fields of science Today, third- and higher-order terms in the remnant spectral phase of the pulses remain a major obstacle for obtaining high-quality few- and single-cycle pulses from in-line post-compression setups. In this Letter, we show how input pulse shaping can successfully be applied to standard post-compression setups to minimize the occurrence of high-order phase components during nonlinear propagation and to directly obtain pulses with durations down to 3 fs. Furthermore, by combining this pulse shaping of the input pulse with new-generation broadband chirped mirrors and material addition for remnant third-order phase correction, pulses down to $2.2 \mathrm{fs}$ duration have been measured. (2018 Optical Society of America

OCIS codes: (320.5540) Pulse shaping; (320.7110) Ultrafast nonlinear optics; (320.7090) Ultrafast lasers; (320.7100) Ultrafast measurements.

https://doi.org/10.1364/OL.43.000337

The generation of few- and single-cycle pulses has been experiencing increasing interest due to its multiple applications in diverse fields, e.g., high-order harmonic generation (HHG) [1], ultrafast characterization of solids [2,3], ultrafast spectroscopy techniques [4], the driving and probing of electronic currents in materials at multi-petahertz frequencies [5], and compact setups for accelerating electrons to $\mathrm{MeV}$ energies at $1 \mathrm{kHz}$ repetition rates [6].

The most common technique is based on gas-filled hollowcore fiber (HCF) post-compression [7], an adaptation to the millijoule energy level of a technique previously demonstrated in optical fibers [8]. Under this scheme, the pulse spectrum is first broadened by nonlinear light-matter interaction during propagation. Afterwards, the outcoming pulse spectral phase (mainly second- and third-order phase terms arising from linear and nonlinear propagation) must be compensated for by introducing adequate amounts of group-delay dispersion (GDD) and third-order dispersion (TOD). Chirped mirrors (CMs), pulse compressors (e.g., based on gratings, prisms, or grisms), or dispersive media are typically used in in-line configurations (i.e., where the beam propagates without splitting at interferometers or delay lines), for achieving post-compressed pulses down to the few-cycle regime in the visible and near-infrared wavelength range [9-13]. Finding the optimum spectral phase compensation stands as the last issue to obtain further compression. In a spectral phase compensation approach alternative to the in-line setups, known as coherent pulse synthesis [14,15], the beam is split into different wavelength channels; its phase is compensated for by diverse CM sets adapted to each spectral band and, finally, the channels are recombined. This technique allows obtaining sub-cycle pulses, although, due to its complexity, it is quite exclusive, and few laboratories can use it.

According to the results systematically reported by laboratories around the world, the ultimate post-compression with in-line spectral phase compensation schemes is mostly limited by the residual third-order phase arising from the nonlinear process [9-12,16-22]. This issue has been addressed by using materials with high TOD/GDD ratios, such as water [10,19], z-cut potassium dihydrogen phosphate (KDP) [17], and z-cut ammonium dihydrogen phosphate (ADP) [12], which have enabled obtaining pulses with full-width at-half-maximum (FWHM) durations around 3 fs.

In this Letter, we first present an alternative approach, where the input pulse is shaped before entering the HCF in order to alter the nonlinear process and to pre-compensate for the remnant third-order phase after nonlinear propagation. We show that this approach enables the direct generation of nearsingle-cycle output pulses in both Ne- and Ar-filled HCFs. Thus, input pulse shaping is shown to be a flexible tool for controlling remnant higher-order spectral phase terms. In 
the second part of this Letter, we present what is, to the best of our knowledge, the best achievable pulse compression using in-line setups by combining the nonlinear process control via pulse shaping with new-generation broadband CMs and a high TOD/GDD ratio material (water) in the postcompression stage in order to obtain pulses down to $2.2 \mathrm{fs}$, while maintaining the standard in-line configuration.

In all experiments, pulse characterization was based on the d-scan technique [16], which has been validated and shown to be well-suited for measuring few- and near-single-cycle pulses $[6,9,10-13,17-20,22,23]$. While altering the pulse phase (in our case, by changing material insertion using two glass wedges), the spectra of a nonlinear signal generated by the resulting pulses (here, the second-harmonic generation [SHG], produced in a $5 \mu \mathrm{m}$ thick beta barium borate crystal cut for Type I phase matching) are recorded, thus obtaining a d-scan trace encoding the spectral phase information. Apart from its simplicity and robustness, due to its in-line architecture, one of the main advantages of this technique lies in the intuitiveness of the experimental traces, even prior to applying the reconstruction algorithm. Thus, for instance, a GDD addition to a given pulse produces an overall shift in the $\mathrm{d}$-scan trace along the dispersion axis, TOD gives a trace tilt, and fourth-order dispersion (FOD) gives a quadratic curvature, and so on $[16,24]$. In the particular case of the remnant TOD compensation at the few-cycle postcompression regime, the experimental $\mathrm{d}$-scan trace shows the effect of materials as a change on its tilt $[10,12]$ towards the ideal non-tilted flat trace case, reflecting remnant TOD suppression and near-Fourier-limit compression.

The light source used for the experiments was a chirped pulse amplified (CPA) Ti:sapphire laser system (FemtoPower Compact HE PRO CEP, Femtolasers GmbH) delivering 23.9 fs pulses (FWHM) under optimum compression conditions at a $1 \mathrm{kHz}$ repetition rate with maximum energy of $2.5 \mathrm{~mJ}$ (up to $1.1 \mathrm{~mJ}$ were used in the experiments). The pulse carrier envelope phase (CEP) can be stabilized, with a typical a stability of $100 \mathrm{mrad}$ over $2 \mathrm{~h}$. An acousto-optic device (Dazzler, from Fastlite), placed within the multi-pass amplifier chain, can be used for shaping the pulses prior to the post-compression setup. A $1 \mathrm{~m}$ focal length spherical mirror coupled the amplifier beam into a $1 \mathrm{~m}$ long HCF, with an inner diameter of $250 \mu \mathrm{m}$. The beam pointing was stabilized by feedback-looped piezoelectric controlled positioners. The HCF was filled with gas (Ar or $\mathrm{Ne}$ ) at different pressures, and the output pulse spectral phase was compensated for by means of CMs and glass wedges. Two different sets of CMs have been used in the experiments. The first set (CM1) was used for few-cycle pulse post-compression, presenting a nominal GDD of $-40 \mathrm{fs}^{2} /$ pair at $800 \mathrm{~nm}$ and a minimum reflectance of $99 \%$ from 500 to $1050 \mathrm{~nm}$ (PC70, Ultrafast Innovations $\mathrm{GmbH})$. The second set (CM2) was used for compressions down to the single-cycle regime and presents improved bandwidth specifications in the region of interest, including the blue range, with a nominal GDD of $-40 \mathrm{fs}^{2} /$ pair at $800 \mathrm{~nm}$ and a minimum reflectance of $99 \%$ from 450 to 1000 nm (PC1332, Ultrafast Innovations GmbH).

In order to explore the capability of controlling the output pulse spectral phase after the nonlinear process by means of input pulse shaping, we have scanned a range of dispersion parameters, namely GDD (ranging from $-200 \mathrm{fs}^{2}$ to $681 \mathrm{fs}^{2}$ ), TOD (from $-23,541 \mathrm{fs}^{3}$ to $45,000 \mathrm{fs}^{3}$ ), and FOD (from $-65,000 \mathrm{fs}^{4}$ to $\left.111,000 \mathrm{fs}^{4}\right)$ at different gas pressure conditions (ranging from 0.1 to 1.3 bars for Ar and from 0.7 to 2.4 bars for $\mathrm{Ne}$ ), where the 0 value in the GDD, TOD, and FOD refers to those applied for obtaining the shortest pulses (23.9 fs) from the CPA chain at the HCF entrance.

Figure 1 shows how three different input pulse conditions affect the output pulse structure, while keeping the other
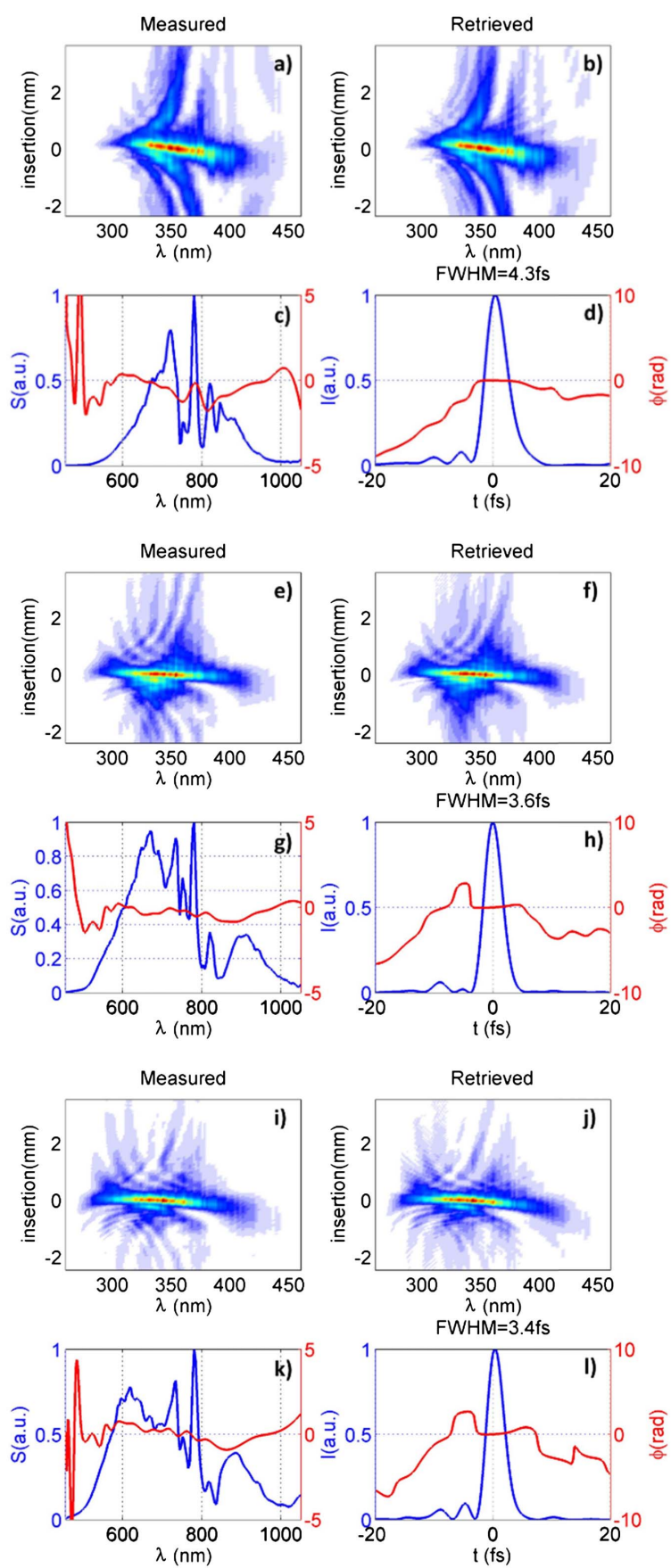

Fig. 1. Pulse post-compression at Ne-filled HCF with (a)-(d) $0 \mathrm{fs}^{2}$, $0 \mathrm{fs}^{3}$, and $0 \mathrm{fs}^{4}$ input pulses; (e)-(h) $187 \mathrm{fs}^{2}, 0 \mathrm{fs}^{3}$, and $0 \mathrm{fs}^{4}$ input pulses; and (i)-(l) $106 \mathrm{fs}^{2}, 3000 \mathrm{fs}^{3}$, and $0 \mathrm{fs}^{4}$ input pulses. (a), (e), and (i) label the corresponding SHG d-scan experimental traces; (b), (f), and (j) label the retrieved traces; (c), (g), and (k) label the experimental fundamental spectra (blue curves) and retrieved spectral phases (red curves); and (d), (h), and (l) the reconstructed pulse temporal intensities (blue curves) and temporal phases (red curves). 
experimental parameters constant. We present four plots for each input parameter: the measured $\mathrm{d}$-scan trace $(\mathrm{a}, \mathrm{e}, \mathrm{i})$, the reconstructed $d$-scan trace $(b, f, j)$, the measured spectrum and retrieved phase (c, g, k) and the temporal pulse structure $(d, h, l)$, obtained for the wedge insertion yielding the minimum pulse duration. Figures $1(\mathrm{a})-1(\mathrm{~d})$ correspond to the result obtained using $\mathrm{Ne}$ with an input pulse at $\left(0 \mathrm{fs}^{2}, 0 \mathrm{fs}^{3}, 0 \mathrm{fs}^{4}\right)$ conditions and no remnant TOD compensation after the nonlinear process (i.e., no material with high TOD/GDD ratio was used). Results with pulse duration around $4.3 \mathrm{fs}$ (FWHM) are in agreement with the cases already known in the literature [9-13], presenting a negative remnant third-order phase, as shown by the $\mathrm{d}$-scan trace tilt [Fig. 1(a)] and the resulting pre-pulses [Fig. 1(d)].

A first approach to adapt the input pulse phase to improve the post-compression was to change the GDD of the input pulse. After performing a scan on GDD, we observed a minimum duration where $187 \mathrm{fs}^{2}$ were added to the input pulse spectral phase. The results obtained under these new conditions are presented in Figs. 1(e)-1(h). Note that the wedge material insertion to obtain the optimum output pulse may be different from the previous case. The addition of GDD contributes to a broader spectrum presenting less remnant TOD [note that the tilt of the d-scan trace has decreased in Fig. 1(e) compared to Fig. 1(a)]. With these new input pulse conditions and wedge insertion after the HCF, the output pulse duration has decreased to 3.6 fs (FWHM).

Further improvement can be obtained when higher-order dispersion components are used. Figures 1(i)-1(l) present the corresponding results for these conditions: $\mathrm{GDD}=106 \mathrm{fs}^{2}$, $\mathrm{TOD}=3000 \mathrm{fs}^{3}$, and $\mathrm{FOD}=0 \mathrm{fs}^{4}$. Now the optimal post-compressed pulse has a duration of $3.4 \mathrm{fs}$ (FWHM). It still presents a negative remnant third-order phase, so shorter durations and higher pulse quality/contrast may be achieved if the pulse is better compressed, as we will discuss later. In any case, we can conclude that the control of the input pulse phase allows directly obtaining pulses with temporal durations close to 3.4 fs from Ne-filled HCF compressors.

Figure 2 summarizes the experimental output pulse durations (FWHM) obtained when scanning the input pulse TOD for the HCF filled with Ar at 0.4 bars (circles) and $\mathrm{Ne}$ at 2 bars (asterisks). As expected from previous works $[25,26]$, adding positive TOD to the input pulse allows us

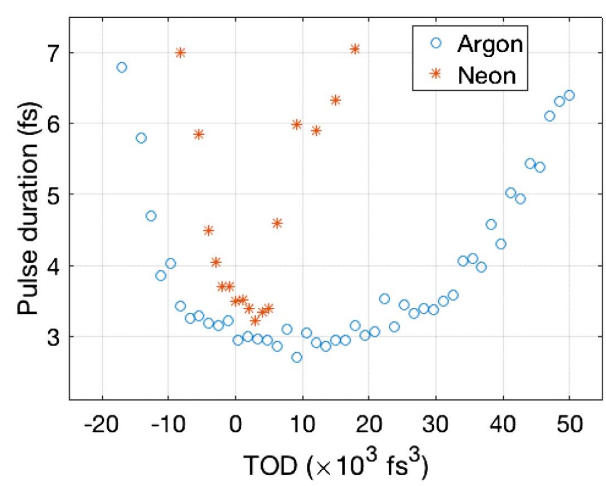

Fig. 2. Post-compressed pulse duration (FWHM) applying TOD components to the input pulse $\left(\mathrm{GDD}=106 \mathrm{fs}^{2}\right)$ with the HCF filled with $\mathrm{Ar}$ at 0.4 bars (circles) and $\mathrm{Ne}$ at 2 bars (asterisks).

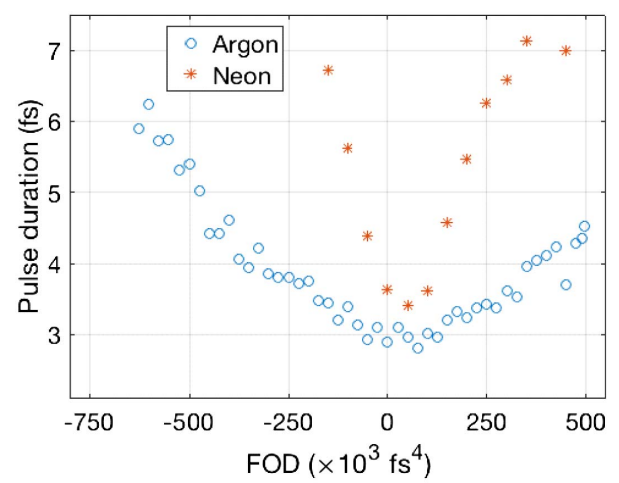

Fig. 3. Post-compressed pulse duration (FWHM) applying FOD components to the input pulse $\left(\mathrm{GDD}=106 \mathrm{fs}^{2}\right.$, TOD $=0 \mathrm{fs}^{3}$ ) with the HCF filled with Ar at 0.4 bars (circles) and $\mathrm{Ne}$ at 2 bars (asterisks).

to obtain shorter pulses (minimum duration with $\mathrm{Ne}$ is $3.4 \mathrm{fs}$, for $\mathrm{TOD}=3000 \mathrm{fs}^{3}$ and with $\mathrm{Ar}, 2.7 \mathrm{fs}$, for TOD $=9167 \mathrm{fs}^{3}$ ), since the negative TOD from the nonlinear process, mainly the self-steepening, is compensated for. While the $\mathrm{Ne}$ cases present longer post-compressed pulse durations compared to the Ar cases, due to a lower nonlinearity at those conditions, the latter are less sensitive to the variation of the input pulse TOD. This would be in agreement with Ref. [25], exhibiting a lower TOD sensitivity for higher B-integral values.

In a similar way, Fig. 3 shows the experimental output pulse durations (FWHM) for different input pulse FOD values for both Ne-filled (asterisks) and Ar-filled (circles) HCF. The addition of a certain positive FOD to the input pulse allows us to get shorter pulses (3.4 fs with $\mathrm{Ne}$ for $\mathrm{FOD}=5000 \mathrm{fs}^{4}$, and $2.8 \mathrm{fs}$ with $\mathrm{Ar}$ for FOD $\left.=7500 \mathrm{fs}^{4}\right)$. As observed with the TOD, the post-compression with $\mathrm{Ne}$ is more sensitive to the FOD than the Ar one.

Previously, we mentioned that some of the obtained pulses may be further compressed by compensating for the residual phase. For exploring these limits, the CM2 set was used. Compared to CM1, the CM2 set presents a broader operating spectral range, shifted towards the short wavelengths, which allows including the higher frequencies of the broadened spectrum. Using $420 \mu \mathrm{J}$ pulses and an HCF filled with Ar at a pressure of 409 mbars, we have observed that the remnant third-order phase can be almost compensated for by pulse shaping $\left(\mathrm{GDD}=-150 \mathrm{fs}^{2}\right.$, TOD $=-500 \mathrm{fs}^{3}$, and FOD $=$ 150, $\left.000 \mathrm{fs}^{4}\right)$. Additional TOD compensation was provided by placing a $2 \mathrm{~mm}$ long water cell, as proposed and demonstrated in [10]. Ultimate post-compression was achieved, which has resulted in 2.2 fs FWHM pulses in the case shown in Fig. 4. The degree of phase compensation, taking into account the available spectrum, is very high, since the Fourier-transform limit (FTL) is $2 \mathrm{fs}$. Moreover, the retrieved pulse peak intensity corresponds to $76.8 \%$ of the corresponding FTL pulse. Output energy is $100 \mu \mathrm{J}$. The retrieved pulse envelope reconstruction demonstrates that the optimally compressed $2.2 \mathrm{fs}$ pulses are single-cycle ( 1.04 cycles, calculated as the temporal phase variation, in units of $2 \pi$, within the pulse intensity FWHM) which, to the best of our knowledge, is also the shortest pulse duration achieved in an in-line HCF post-compression setup. This 

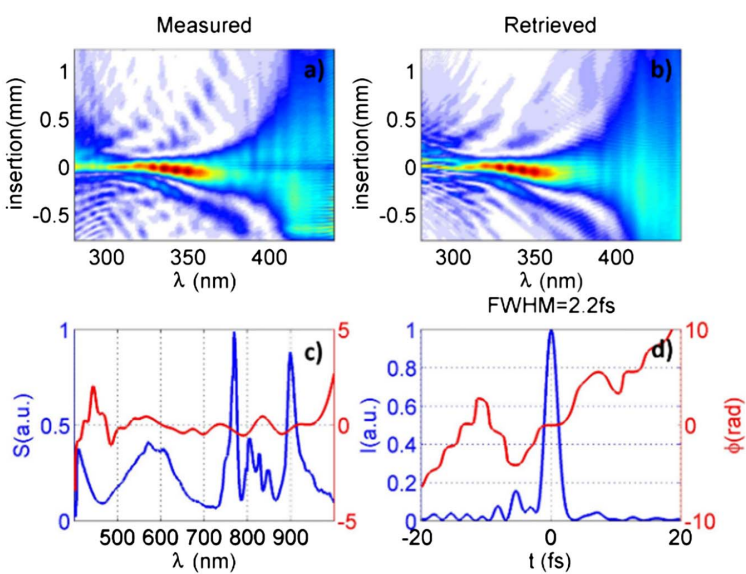

Fig. 4. (a) SHG d-scan experimental trace; (b) retrieval trace; (c) experimental fundamental spectrum, blue line, and retrieved spectral phase, red line; and (d) reconstructed pulse for a post-compressed pulse in an Ar-filled HCF ( $P=409$ mbars) with the shaping of the input pulse, using the CM2 set for the main spectral phase compensation, and after propagation in a $2 \mathrm{~mm}$ thick water-filled cell in order to further minimize the residual third-order phase generated during nonlinear propagation.

result is reproducible, since we performed eight independent measurements at the same conditions, which gave an average duration of $2.274 \mathrm{fs}$ with a standard deviation of $0.084 \mathrm{fs}$.

To conclude, we have shown that input pulse shaping can be used to alter and control the nonlinear propagation within gas-filled HCFs used for in-line post-compression setups. The post-compression can be improved with respect to the nonshaped case by adapting the input pulse to obtain an optimized response both in spectrum and phase, achieving compression down to the $3 \mathrm{fs}$ level, even through a manual routine, optimizing the $\mathrm{d}$-scan trace by acting on spectral phase orders (GDD, TOD, or FOD) alternatively. Results in Ar and $\mathrm{Ne}$ support the robustness of this scheme. Furthermore, we demonstrate ultimate compression down to single-cycle pulses by synergic combination of input pulse shaping, enhanced broadband CMs, and further TOD compensation (in this case, by propagation in a $2 \mathrm{~mm}$ long water cell).

Using this combination of optimization techniques, highquality pulses down to $2.2 \mathrm{fs}$ were obtained, while the FTL of the spectrum was $2.0 \mathrm{fs}$. Therefore, this hybrid strategy grants access to the generation of intense pulses in the single-cycle regime, based on the standard in-line post-compression setups available at most ultrashort pulse laboratories today, and with direct application, e.g., as high-resolution probes in experiments on atomic and molecular dynamics, or as driving pulses for direct generation (intensity gating) of isolated attosecond pulses by means of HHG.

Funding. Ministerio de Economía y Competitividad (MINECO) (FIS2013-44174-P, FIS2015-71933-REDT, FIS2016-75652-P, FIS2017-87970-R); Consejería de Educación, Junta de Castilla y León (SA046U16, SA116U13); Fundação para a Ciência e a Tecnologia (FCT) (M-ERANET2/0002/2016); European Regional Development Fund (ERDF) (NORTE-01-0145-FEDER-022096).
Acknowledgment. The authors thank the staff of the Centro de Láseres Pulsados (CLPU) for granting access to its facilities.

\section{REFERENCES}

1. A. Blättermann, C. Ott, A. Kaldun, T. Ding, V. Stooß, M. Laux, M. Rebholz, and T. Pfeifer, Opt. Lett. 40, 3464 (2015).

2. A. Schiffrin, T. Paasch-Colberg, N. Karpowicz, V. Apalkov, D. Gerster, S. Mühlbrandt, M. Korbman, J. Reichert, M. Schultze, S. Holzner, J. V. Barth, R. Kienberger, R. Ernstorfer, V. S. Yakovlev, M. I. Stockman, and F. Krausz, Nature 493, 70 (2013).

3. M. Schultze, E. M. Bothschafter, A. Sommer, S. Holzner, W. Schweinberger, M. Fiess, M. Hofstetter, R. Kienberger, V. Apalkov, V. S. Yakovlev, M. I. Stockman, and F. Krausz, Nature 493, 75 (2013).

4. J. Liu, K. Okamura, Y. Kida, T. Teramoto, and T. Kobayashi, Opt. Express 18, 20645 (2010).

5. M. Garg, M. Zhan, T. T. Luu, H. Lakhotia, T. Klostermann, A. Guggenmos, and E. Goulielmakis, Nature 538, 359 (2016).

6. D. Guénot, D. Gustas, A. Vernier, B. Beaurepaire, F. Böhle, M. Bocoum, M. Lozano, A. Jullien, R. Lopez-Martens, A. Lifschitz, and J. Faure, Nat. Photonics 11, 293 (2017).

7. M. Nisoli, S. DeSilvestri, and O. Svelto, Appl. Phys. Lett. 68, 2793 (1996).

8. R. L. Fork, C. H. Brito Cruz, P. C. Becker, and C. V. Shank, Opt. Lett. 12, 483 (1987).

9. F. Böhle, M. Kretschmar, A. Jullien, M. Kovacs, M. Miranda, R. Romero, H. Crespo, U. Morgner, P. Simon, R. Lopez-Martens, and T. Nagy, Laser Phys. Lett. 11, 95401 (2014).

10. F. Silva, M. Miranda, B. Alonso, J. Rauschenberger, V. Pervak, and H. Crespo, Opt. Express 22, 10181 (2014).

11. D. Fabris, W. Holgado, F. Silva, T. Witting, J. W. G. Tisch, and H. Crespo, Opt. Express 23, 32803 (2015).

12. H. Timmers, Y. Kobayashi, K. F. Chang, M. Reduzzi, D. M. Neumark, and S. R. Leone, Opt. Lett. 42, 811 (2017).

13. W. Holgado, C. Hernández-García, B. Alonso, M. Miranda, F. Silva, O. Varela, J. Hernández-Toro, L. Plaja, H. Crespo, and I. J. Sola, Phys. Rev. A 95, 63823 (2017).

14. A. Wirth, M. T. Hassan, I. Grguras, J. Gagnon, A. Moulet, T. T. Luu, S. Pabst, R. Santra, Z. A. Alahmed, A. M. Azzeer, V. S. Yakovlev, V. Pervak, F. Krausz, and E. Goulielmakis, Science 334, 195 (2011).

15. M. Hassan, T. Luu, A. Moulet, and O. Raskazovskaya, Nature 530, 66 (2016).

16. M. Miranda, T. Fordell, C. Arnold, A. L'Huillier, and H. Crespo, Opt. Express 20, 688 (2012).

17. M. Miranda, J. Penedones, C. Guo, A. Harth, M. Louisy, L. Neoričić, A. L'Huillier, and C. L. Arnold, J. Opt. Soc. Am. B 34, 190 (2017).

18. C. M. Heyl, H. Coudert-Alteirac, M. Miranda, M. Louisy, K. Kovacs, V. Tosa, E. Balogh, K. Varjú, A. L'Huillier, A. Couairon, and C. L. Arnold, Optica 3, 75 (2016).

19. M. Louisy, C. L. Arnold, M. Miranda, E. W. Larsen, S. N. Bengtsson, D. Kroon, M. Kotur, D. Guénot, L. Rading, P. Rudawski, F. Brizuela, F. Campi, B. Kim, A. Jarnac, A. Houard, J. Mauritsson, P. Johnsson, A. L'Huillier, and C. M. Heyl, Optica 2, 563 (2015).

20. A. Tajalli, B. Chanteau, M. Kretschmar, H. G. Kurz, D. Zuber, M. Kovačev, U. Morgner, and T. Nagy, Opt. Lett. 41, 5246 (2016).

21. B. E. Schmidt, P. Béjot, M. Giguère, A. D. Shiner, C. Trallero-Herrero, É. Bisson, J. Kasparian, J.-P. Wolf, D. M. Villeneuve, J.-C. Kieffer, P. B. Corkum, and F. Légaré, Appl. Phys. Lett. 96, 121109 (2010).

22. E. Conejero-Jarque, J. San Roman, F. Silva, R. Romero, W. Holgado, M. A. Gonzalez-Galicia, B. Alonso, I. Sola, H. Crespo, E. C. Jarque, J. S. Roman, F. Silva, R. Romero, W. Holgado, M. A. Gonzalez-Galicia, I. Sola, and H. Crespo, "Universal route to optimal few- to single-cycle pulse generation in hollow-core fiber compressors," arXiv:1704.01744 (2017).

23. B. Alonso, M. Miranda, F. Silva, V. Pervak, J. Rauschenberger, J. San Roman, I. J. Sola, and H. Crespo, Appl. Phys. B 112, 105 (2013).

24. V. Loriot, G. Gitzinger, and N. Forget, Opt. Express 21, 24879 (2013).

25. A. Suda and T. Takeda, Appl. Sci. 2, 549 (2012).

26. G. Fan, T. Balčiūnas, T. Kanai, T. Flöry, G. Andriukaitis, B. E. Schmidt, F. Légaré, and A. Baltuška, Optica 3, 1308 (2016). 